\title{
Human FAT1 cadherin controls cell migration and invasion of oral squamous cell carcinoma through the localization of $\beta$-catenin
}

\author{
YUKIKO NISHIKAWA ${ }^{1}$, TATSUHIKO MIYAZAKI ${ }^{2,3}$, KOH-ICHI NAKASHIRO $^{1,4}$, HIDEHISA YAMAGATA $^{5}$, \\ MAYUMI ISOKANE ${ }^{1}$, HIROYUKI GODA ${ }^{1}$, HIROSHI TANAKA ${ }^{1}$, RYOTA OKA ${ }^{1}$ and HIROYUKI HAMAKAWA ${ }^{1,4}$ \\ Departments of ${ }^{1}$ Oral and Maxillofacial Surgery, and ${ }^{2}$ Pathogenomics, Ehime University Graduate \\ School of Medicine; Departments of ${ }^{3}$ Immunopathology, and ${ }^{4}$ Growth and Tumor Regulation, \\ Ehime Proteo-Medicine Research Center, Ehime University, 454 Shitsukawa, To-on, Ehime 791-0295; \\ ${ }^{5}$ Education and Training Center, Kansai Rosai Hospital, Inabaso 3-1-69, Amagasaki, Hyogo 660-8511, Japan
}

Received November 30, 2010; Accepted January 28, 2011

DOI: 10.3892/or.2011.1324

\begin{abstract}
FAT1 [Homo sapiens FAT tumor suppressor homolog 1 (Drosophila)] is an intrinsic membrane protein classified as a member of the cadherin superfamily. The FATl gene is a tumor suppressor in humans as well as being the pivotal gene for cell morphogenesis and migration. Deletion of this gene could play a role in the characteristics of oral squamous cell carcinomas (OSCCs), involving cell adhesion, migration and/ or invasion. This study investigated the mechanisms by which FAT1 is involved in the biological behavior of OSCCs. First, a rat monoclonal antibody was developed against a FAT1 intracellular domain epitope, and used for an immunohistochemical study of FAT1 in clinically obtained OSCC samples. FAT1 was localized at lamellipodial edges or cell-cell boundaries in normal cells and well differentiated OSCCs, but showed a diffuse cytoplasmic and nuclear distribution in moderatelypoorly differentiated OSCCs. FAT1-siRNA was transfected into OSCCs resulting in a drastic inhibition of cell migration and invasion based on the suppression of FAT1 expression and disorganized localization of $\beta$-catenin which is associated with cell polarity and migration. These results suggested that FAT1 may be involved in the migration and invasion mechanisms of OSCCs and, therefore, it could be an important target for the development of new therapeutic strategies.
\end{abstract}

\section{Introduction}

The gene product of FAT1 [Homo sapiens FAT tumor suppressor homolog 1 (Drosophila)] is an intrinsic membrane

Correspondence to: Dr Tatsuhiko Miyazaki, Department of Pathogenomics, Ehime University Graduate School of Medicine, 454 Shitsukawa, To-on, Ehime, 791-0295, Japan

E-mail:miyazaki@m.ehime-u.ac.jp

Key words: FAT1, $\beta$-catenin, tumor invasion, siRNA, monoclonal antibody, oral squamous cell carcinoma protein with huge molecular weight $(\sim 500 \mathrm{kD})$ which is classified as a member of cadherin superfamily. This gene product (hFAT1 protocadherin: FAT1) regulates actin dynamics, cell to cell contact and cell polarity and associate with Ena/VASP, Homer 3, and $\beta$-catenin (1-3). On the other hand, FAT1 is involved in the structure and function of the glomerular slit by podocytes (4), and the development of the brain as well as its function, especially regarding its association with the susceptibility to bipolar affective disorder $(5,6)$.

Importantly, FAT1 is also considered to be a tumor suppressor gene (7). Since human FATl was cloned as a homologue of a tumor suppressor gene in Drosophila (1), there have been no studies that focused on the tumor suppressor function or investigated the clinical associations of this gene. A previous study reported the homozygous loss of FAT1 in OSCCs detected by a CGH-array (8), followed by another study describing that the loss of heterozygosity of FAT1 might be an important factor in the development of astrocytic tumors (9). However, the mechanism involved in how it suppresses such carcinogenesis has not yet been clarified.

The cytoplasmic domain of FAT1 binds to $\beta$-catenin and plays a pivotal role in cell migration, polarization and morphogenesis $(3,10-16)$. Therefore, the deletion of this gene could implicate the characteristics and biological behavior of OSCCs that involve cell adhesion, migration and/or invasion, which might result in poor prognosis. It is worthwhile to elicit the role of this gene product with regard to the mechanism of cell migration and/or invasion, especially the association with $\beta$-catenin to possibly identify a new therapeutic strategy to prevent the invasive growth of OSCCs. The current study demonstrated the expression and localization pattern of FAT1 in OSCC cases as well as in OSCC cell lines, simultaneously revealing a change of the morphological characteristics and biological behavior of OSCCs in response to the RNA silencing of FAT1.

\section{Materials and methods}

Development of monoclonal antibodies against FAT1. Rat monoclonal antibodies against human FAT1 were developed 
according to the standard protocol. Briefly, the C-terminus epitope of FAT1 molecule (CLSKKPLEEKPSQPYSARE, AA4345-4363) was chemically synthesized and processed to the immunization. Eight-week-old female WKY/NCrj rats were immunized subcutaneously with $100 \mu \mathrm{g}$ FAT1 epitope and $300 \mu \mathrm{l}$ of complete freund adjuvant (Difco BD, Tokyo, Japan) followed by a booster injection. On day 45, the spleen was dissected and spleen cells were fused with rat myeloma YB2/0 cells. The fused cells were cultured and selected in 96-well microtiter plates then screened by direct ELISA. Selected hybridomas were processed to limiting-dilution cloning then adapted to serum-free culture medium. The supernatant from clone G5 was used in the following experiments after the evaluation of $\mathrm{IgG}$.

Western blot analysis. A Western blot analysis was carried to confirm the monoclonality of anti-FAT1 antibody. Since it was very difficult to extract intact FAT1 from tissue specimen and/or to synthesize whole molecule because of its huge molecular weight, we employed an analysis on the synthesized fragments of FAT1 molecule. The N-terminal fragment FAT1a (aa 22-1034), FAT1b (aa 1035-1982), FAT1c (aa 1982-2920), FAT1d (aa 2921-3991) and C-terminal fragment FAT1e (aa 3992-4578) with a His tag were synthesized by a cell-free protein synthesis system using wheat germ which was equipped with Protemist DTII ${ }^{\circledR}$ (Cell Free Sciences Inc., Yokohama, Japan). The crude and purified protein solutions were separated by electrophoreses and transferred to polyvinylidene difluoride membranes (Millipore, Billerica, MA). The membranes were blocked with 5\% non-fat dried milk (Wako, Osaka, Japan) and 1X TBS-T [25 mM Tris-HCl, $125 \mathrm{mM} \mathrm{NaCl}$, and 0.1\% Tween-20 (Sigma-Aldrich)] overnight at $4{ }^{\circ} \mathrm{C}$. They were then probed with monoclonal rat anti-FAT1 antibodies for $1 \mathrm{~h}$, followed by treatment with horseradish peroxidase-conjugated secondary goat antibodies against rat IgG (Chemicon, Temecula, CA) for $1 \mathrm{~h}$ at room temperature. The immune complexes were visualized with the enhanced chemiluminescence (ECL) Plus kit (GE Healthcare Japan, Tokyo, Japan).

Cells and cell culture. The human OSCC cell lines (HSC2, HSC3, HSC4, OSC2, SAS, B88, KB, SCC9, SCC25, SCC66 and $\mathrm{SCC} 111)(8,17)$ were maintained in Dulbecco's modified Eagle's medium (DMEM) (Wako) supplemented with $10 \%$ fetal bovine serum (FBS; Biosource, Camarillo, CA), $100 \mathrm{U} / \mathrm{ml}$ penicillin, and $100 \mu \mathrm{g} / \mathrm{ml}$ streptomycin (Invitrogen, Carlsbad, CA), and incubated in a humidified atmosphere of $95 \%$ air and $5 \% \mathrm{CO}_{2}$ at $37^{\circ} \mathrm{C}$.

Immunohistochemistry. An immunohistochemical study was performed on OSCC tissue specimens obtained from 52 patients with OSCC at Ehime University Hospital under an appropriate informed consent. Tissue sections which include a portion of the carcinoma lesion as well as a non-tumor area were selected for immunohistochemical analyses. The sections $(4 \mu \mathrm{m})$ of formalin-fixed, paraffin-embedded tissue were deparaffinized and rehydrated, then heated in $10 \mathrm{mM}$ citrate buffer ( $\mathrm{pH} 6.0$ ) for $30 \mathrm{~min}$ in a microwave oven to facilitate antigen retrieval. The rat monoclonal antibody to FAT1, described above, was incubated with the sections overnight. Streptavidin-Biotin method with biotin-conjugated donkey anti-rat IgG (Jackson Immunolaboratory, West Grove, PA) and alkaline phosphatase-conjugated streptavidin was used to amplify the signal. The resulting immunocomplexes were detected with a fuchsin substrate chromogen system (Dako). Simultaneously, the sections were counterstained with hematoxylin, embedded in Ultramount ${ }^{\circledR}$ mounting medium (Dako), then analyzed.

Immunofluorescence. The cells were fixed with $4.0 \%$ paraformaldehyde in phosphate buffer and permeabilized with $0.1 \%$ Triton X-100 in PBS. Non-specific protein binding was blocked with $1 \%$ BSA in PBS, and the cells were incubated with the primary antibodies at the appropriate dilutions. Direct immunofluorescence of FAT1 staining was performed using the Zenon ${ }^{\circledR}$ Alexa Fluor 488 Monoclonal Antibody Labeling $\mathrm{kit}^{\circledR}$ (Molecular Probes, Eugene, OR). Simultaneously double immunofluorescence against $\beta$-catenin was performed using murine anti-human $\beta$-catenin (E5, Santa Cruz Biotechnology, Santa Cruz, CA) and Alexa 647-conjugated Donkey anti-mouse IgG antibody (Jackson Immunolaboratory). The cell nuclei were counterstained by Hoechst $33342^{\circledR}$ (Hoechst, Frankfurt am Main, Germany). Immunofluorescence was examined and captured using a confocal ultra-spectral-microscope system $\mathrm{A} 1^{\circledR}$, (Nikon Instruments, Tokyo, Japan). Post-capture image analyses were performed using the Nikon Confocal software package NIS-Elements $C^{\circledR}$ (Nikon Instruments). Images for presentation were directly obtained from the NIS-Elements C software program.

Design and transfection of synthetic siRNAs. Three pairs of FAT1-specific synthetic siRNAs were designed and synthesized. Target sequences were selected using B-Algo and siPrecise (B-Bridge, Mountain View, CA) for maximum target gene silencing, minimum off-target effects. The sequence of the siRNAs were: sense 1, agggagagguggaggauauTT; antisense 1, auauccuccaccucucccuTT; sense 2, cuacauagagguugagauTT; antisense 2, auccucaaccucuauguagTT; sense 3 cagcaaacccaga agcuauTT; antisense 3, auagcuucuggguuugcugTT. Transfection was performed with Lipofectamine RNAiMax (Invitrogen) mixed with $10 \mathrm{nM}$ siRNA for the assays described below.

Cell proliferation analysis. The cells $\left(5 \times 10^{4}\right)$ were seeded into 60-mm dishes in complete medium with synthetic siRNA and Lipofectamine RNAiMax and incubated for 4 days. The cells were recovered by treatment with $0.05 \%$ trypsin, $0.53 \mathrm{mM}$ EDTA (Wako) and then were counted with a Z1 Coulter counter (Beckman Counter, Fullerton, CA).

RNA isolation. Total cellular RNA was purified from OSCC cells using ISOGEN ${ }^{\circledR}$ (Nippon Gene, Toyama, Japan) according to the manufacturer's instructions. RNA samples from cells treated with siRNA to FAT1, along with an appropriate control, were submitted for analysis. RNA quality was checked by use of the Agilent BioAnalyzer, and samples were profiled only if they had clear distinct $18 \mathrm{~S}$ and $28 \mathrm{~S}$ peaks with no minor peaks present.

Real-time RT-PCR. The relative quantitation of mRNA levels using the comparative CT method ( $\Delta \Delta \mathrm{CT}$ method) was 

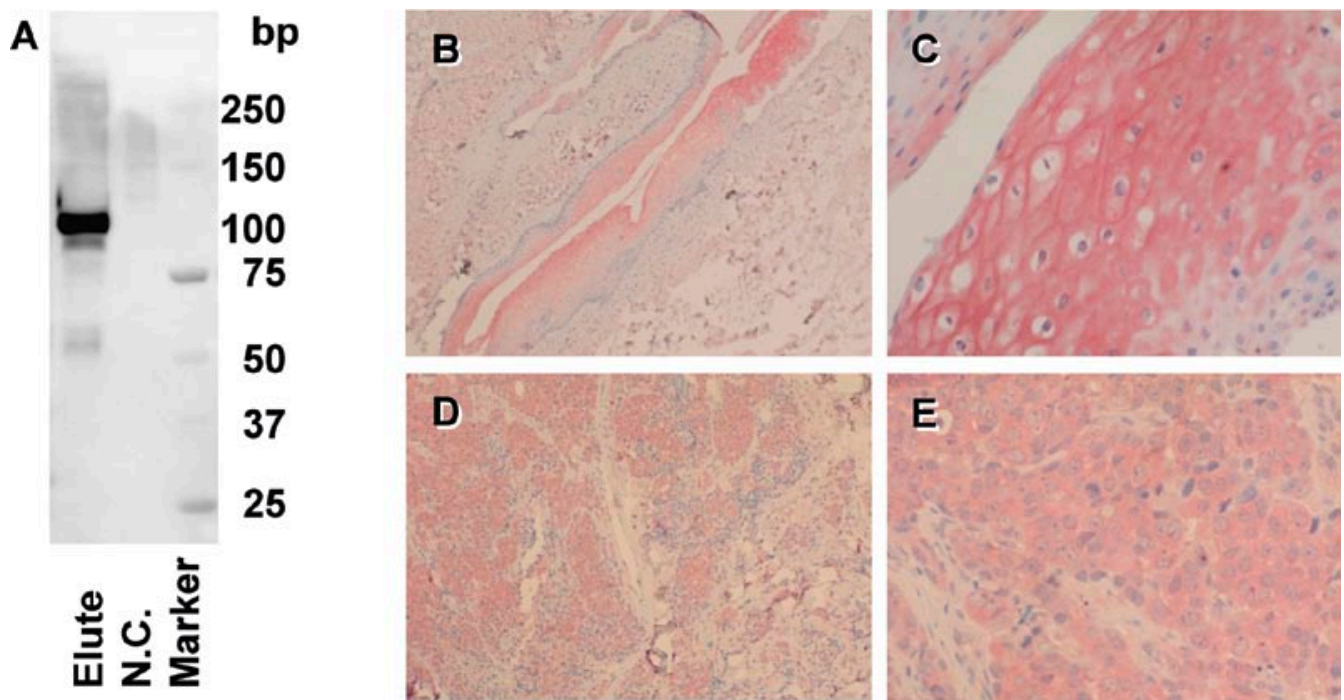

50
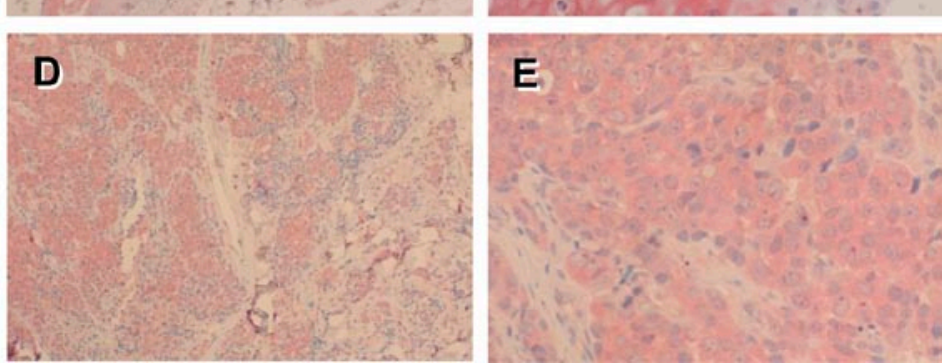

Figure 1. Development of FAT1 specific rat monoclonal antibody and expression of FAT1 protein in OSCC. (A) The rat monoclonal antibody showed a single band in the Western blotting using the C-terminal fragment of FAT1. Lane 1, elute; lane 2, crude solution of negative control. (B and C) Immunohistochemistry against FAT1 intracellular domain epitope in normal tissue, low magnification (B) and high magnification (C). FAT1 positive signals were localized in the cytoplasm as well as at lamellipodial edges or cell-cell boundaries in normal cells. (D and E) Immunohistochemistry to FAT1 in OSCCs that were designated as moderately differentiated squamous cell carcinoma, at low magnification (D) and in high magnification (E). In the OSCC tissue, FAT1 positive signals were seen diffusely in the cytoplasm of OSCCs.

carried out by real-time quantitative RT-PCR, with the One Step SYBR PrimeScript RT-PCR kit II (Takara Bio, Otsu, Japan) in a LightCycler 1.5 System (Roche Diagnostics, Tokyo, Japan). Hydroxymethylbilane synthase (HMBS) mRNA was used as an internal control. Primers used in RT-PCR were: FAT1 sense 1 (GAGTGGTTTCGTCCAAG AGG), FAT1 antisense 1 (TGGAGTCTGGTGGTTGATGA), FAT1 sense 2 (TAGATGGCAACCAAGGAAGC), FAT1 antisense 2 (CGTGAGCGTGTAACCTGAAA), FAT1 sense 3 (TTTTAACCCCGAGTCTGTGC), FAT1 antisense 3 (GCT GTCAGAAGGGGAGTTTG), FAT1 FW (CTGACAAGA GTGCAGGCCACA), and FAT1 RV (AACTGCCCATCA GCAGAGTCAA), HMBS sense (CATGCAGGCTACCAT CCATGTC) and HMBS antisense (GTTACGAGCAGTG ATGCCTACCAA). The $20-\mu 1$ reaction mixture contained: $10 \mu \mathrm{M}$ of forward and reverse primers, $10 \mu \mathrm{l} 2 \mathrm{X}$ One-step SYBR RT-PCR buffer 4 (Takara Bio), $0.8 \mu$ l PrimeScript ${ }^{\circledR}$ One-step enzyme mix 2, $5.6 \mu \mathrm{l}$ RNase Free Water $\mathrm{dH}_{2} \mathrm{O}$, and $2 \mu \mathrm{l}$ total RNA. Reaction conditions were: an RT step at $42^{\circ} \mathrm{C}$ for $5 \mathrm{~min}$ and denaturation at $95^{\circ} \mathrm{C}$ for $10 \mathrm{sec}$, followed by 40 PCR cycles of $95^{\circ} \mathrm{C}$ for $5 \mathrm{sec}, 60^{\circ} \mathrm{C}$ for $20 \mathrm{sec}$. The assay ended with a melting-curve analysis: heating to $95^{\circ} \mathrm{C}$ without a hold, rapid ramping to $65^{\circ} \mathrm{C}$ and holding for $15 \mathrm{sec}$, gradual heating to $95^{\circ} \mathrm{C}$ at $0.1^{\circ} \mathrm{C} / \mathrm{sec}$, and final cooling to $40^{\circ} \mathrm{C}$.

In vitro wound-healing assay. OSCC cells were plated at a high density $\left(1 \times 10^{6}\right.$ cells/well) on a $35-\mathrm{mm}$ collagen-coated glass bottom dish (MatTek. Co., Ashland, MA) and then were cultured for 3 days with or without siRNA against FAT1. Monolayers of the cells were wounded by scraping with the tip of a plastic pipette. Eight hours later, the cells were fixed and stained as indicated above, and then the width of the wound was evaluated using NIS-element software program.
In vitro cell invasion assay. The cell invasion assay was performed using the CytoSelect ${ }^{\mathrm{TM}}$ Cell Migration and Invasion assay kit (Cell Biolabs Inc., San Diego, CA). Briefly, a high density $\left(1 \times 10^{6}\right.$ cells/ml) of OSCC cells with or without siRNA transfection were plated on the inner chamber which had matrix coated $8-\mu \mathrm{m}$ pore with serum-free DMEM then cultured for $24 \mathrm{~h}$. The cells migrated to outer chamber which contained $10 \%$ FBS in DMEM were lysed and quantified with CyQuant ${ }^{\circledR}$ GR fluorescent dye by using a FlexStation III fluorescent microplate reader (Molecular Devices, Tokyo, Japan).

\section{Results}

Expression of FAT1 protein in OSCC. A rat monoclonal antibody to a FAT1 intracellular domain epitope was developed as shown in the Western blot. The C-terminal fragment of FAT1 manifested single band which was labeled by the antibody while the other four fragments did not react with the antibody (Fig. 1A). OSCC tissues from 52 cases were examined by immunohistochemistry for FAT1 expression using this antibody. Immunohistochemistry revealed 50 out of 52 cases were FAT1 positive at various levels and manifested a staining pattern of FAT1 in cytoplasm as well as at the edge of lamellipodia or cell-cell boundaries in normal cells (Fig. 1B and C) and well differentiated OSCCs. On the other hand, FAT1 expression showed a diffuse cytoplasmic and nuclear pattern in moderately-poorly OSCCs (Fig. 1D and E). FAT1 was expressed in OSCCs, as it is in a variety of tissues but the localization of intracytoplasmic domain of FAT1 differed between OSCC and benign tissue specimen.

Expression of FAT1 in OSCC cell lines. The expression of FAT1 mRNA was examined in human OSCC cell lines by RT-PCR. The expression of FAT1 mRNA was detected in 
A FAT1 mRNA expression
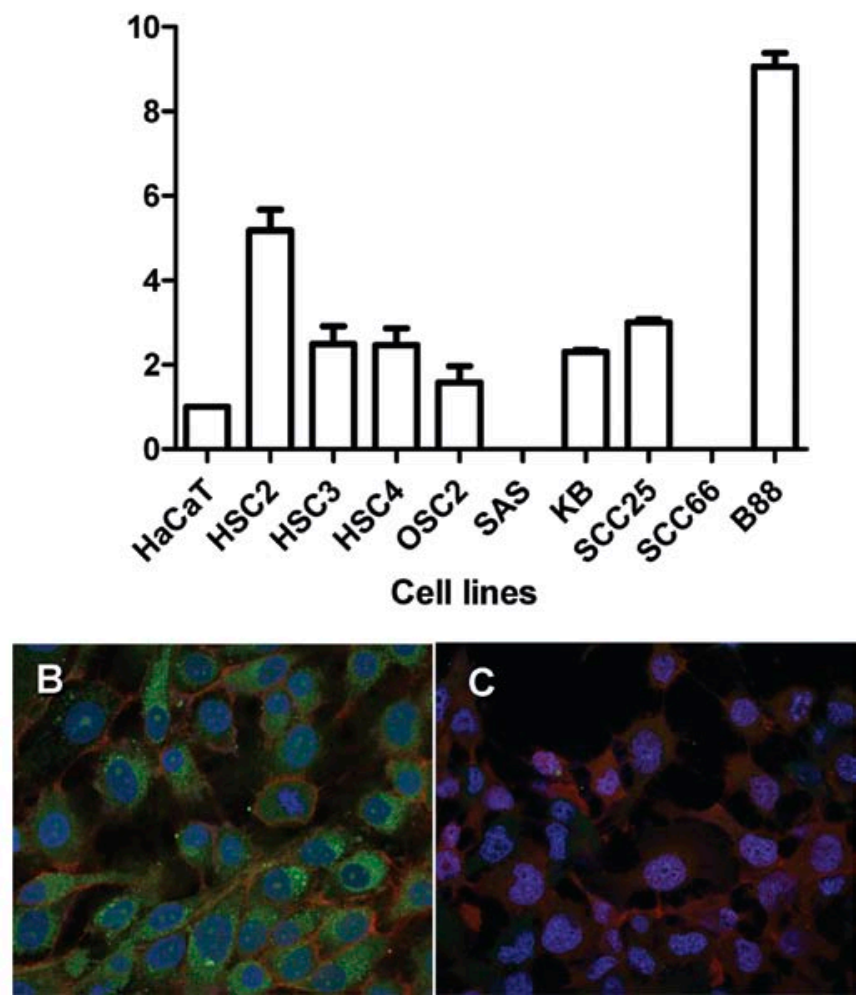

Figure 2. Expression of FAT1 in the OSCC cell lines. (A) In OSCCs, expression of FAT1 mRNA was detected in most of the cell lines except for SAS and SCC66. (B and C) Double immunofluorescence to FAT1 (Alexa-488, green) and $\beta$-catenin (Alexa-647, red). HSC2 showed positive staining for FAT1 in the cytoplasm and at the lamellipodial edges or cell-cell boundaries as well as for $\beta$-catenin at the lamellipodial edges or on the cell membrane with cell to cell adhesion (B). SAS showed the $\beta$-catenin accumulated in the cytoplasm and nuclei in FAT1-lacking SAS without cell to cell adhesion, thus resulting in a poorly differentiated shape of the cells (C).

all the cell lines at various levels except for SAS and SCC66 (Fig. 2A). Subsequently, immunofluorescence was performed on those cultured cells and obtained similar result. A double immunofluorescence analyses was conducted on several kinds of FAT1-positive and -negative cell lines including HSC2, HSC3, B88 (positive) and SAS (negative). Those double immunofluorescence studies revealed that $\beta$-catenin was localized along the cell membrane in FAT1-expressing OSCCs and cell to cell adhesion was consistent with the morphological features of squamous epithelium (Fig. 2B), whereas $\beta$-catenin accumulated in the cytoplasm and nuclei in SAS cells lacking FAT1 without cell to cell adhesion, thus resulting in a poorly differentiated shape of the cells (Fig. 2C).

FAT1 RNAi effects on cell proliferation and morphology of OSCCs. RNAi was used to clarify the role of FAT1 in cell proliferation as well as in the morphological change of OSCCs. In the first experiment we examined whether OSCC can grow under FAT1 suppression. The transfection of $10 \mathrm{nM}$ siRNA of each synthetic siFAT1s markedly reduced the expression of the FAT1 mRNA at 24 and $48 \mathrm{~h}$ after transfection (Fig. 3A), and showed a tendency to upregulate cell growth while it was not significant (data not shown). Double immunofluorescence of FAT1 and $\beta$-catenin, showed that the
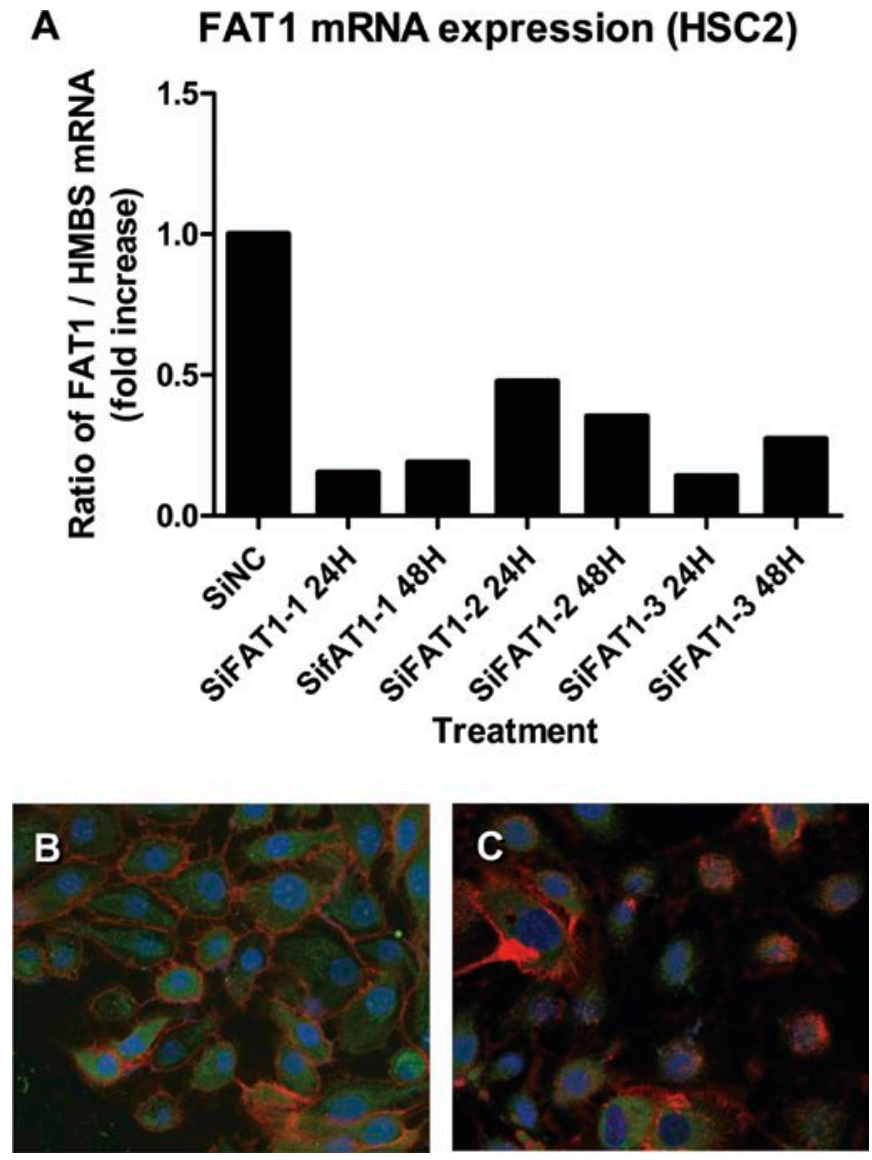

Figure 3. RNAi effects of siFAT1s in human OSCC. (A) siFAT1s (10 nM) were transfected into the OSCC with Lipofectamine RNAiMax. The effect of RNAi on FAT1 mRNA expression was evaluated by RT-PCR. Three siRNAs suppressed the expression of FAT1 mRNA in the cells both at 24 and at $48 \mathrm{~h}$ after transfection. (B and C) Double immunofluorescence to FAT1 (5) and $\beta$-catenin (18). At $48 \mathrm{~h}$ after transfection, in control HSC 2 cells (B), $\beta$-catenin was expressed neatly on the cell membrane whereas in FAT1 knockdown cells, vague and diffuse signal of $\beta$-catenin was observed in the cytoplasm and around the nuclei (C).

control HSC2 exhibited positive staining for both FAT1 and $\beta$-catenin with polarity and localization at the lamellipodial edge and on the cell membrane, whereas siFAT1 transfected HSC2 lacked FAT1 positivity and showed the staining of $\beta$-catenin to have accumulated in both the cytoplasm and nucleus (Fig. 3B and C).

FAT1 RNAi effects on cell migration/invasion. The expression of FAT1-altered cell mobility was next evaluated using a classical wound-healing assay. We observed a significant decrease in the migration ability of siFAT1 transfected HSC2 in comparison to the control HSC2 cells (Fig. 4A) as well as in HSC3 and B88 cells, respectively (data not shown). Control cells migrated into the wound space in an organized manner with observable polarity, so that $\beta$-catenin localized at the apical side (toward the wound, Fig. 4B and C), whereas siFAT1 transfected cells manifested limited migration into the wound space in a disorganized manner with a vague polarity of the individual cells (Fig. 4D and E).

A cell invasion assay of OSCC cells was conducted, including $\mathrm{HSC} 2$ which was detected by the fluorescence level of lysed migrated cells labeled by CyQuant dye. The assay 
A
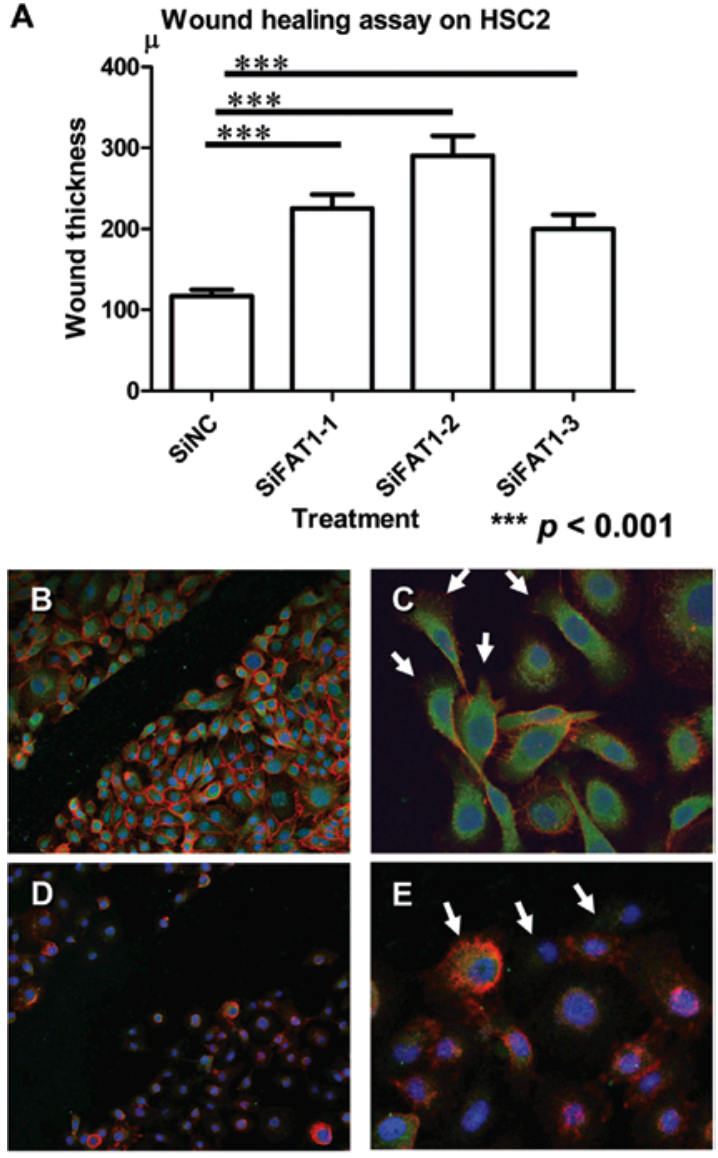

F

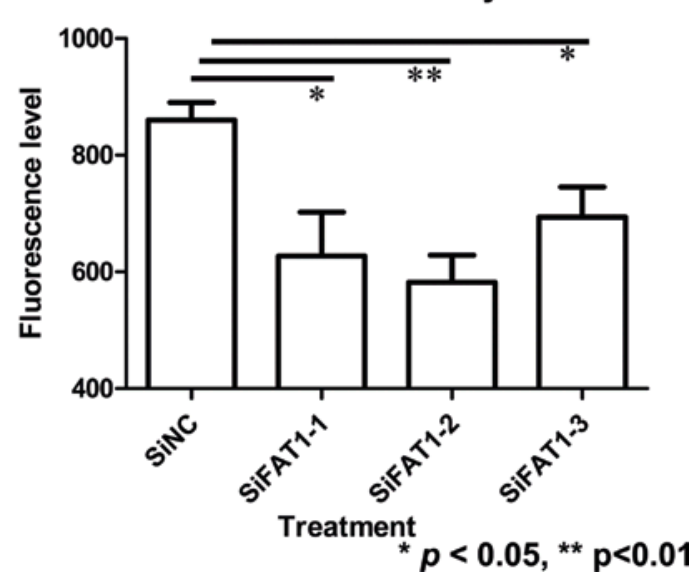

Figure 4. RNAi effects of siFAT1s in wound healing assay and trans-well invasion assay of human OSCCs. (A) Representative results of wound thickness at $8 \mathrm{~h}$ after scratch in the wound healing assay using HSC2 OSCC cell line with or without siFAT1 transfection. All of transfected cells manifested significantly larger wound thickness in comparison to mock transfected control cells. (B-E) Double immunofluorescence to FAT1 (5) and $\beta$-catenin (18). (B) Mock transfected cells shows thinner wound space with organized cell migration with cytoplasmic FAT1 positive signals as well as those of $\beta$-catenin localized in cell borders while siFAT1 transfected cells. (D) Thicker wound space with disorganized cell migration with very limited FAT1-positive signals and vaguely distributed $\beta$-catenin signals. (C) In higher magnification, $\beta$-catenin localized at the lamellipodial edge in the apical side (arrows) toward wound space, nevertheless the siFAT1 transfected cells (E) show no significant localization of $\beta$-catenin at the apical end (arrows). (F) Representative result of cell migration/invasive assay using siFAT1 transfected and mock transfected HSC2 OSCCs. The fluorescence levels reflecting migrated cell numbers showed a significant decrease in siFAT1 transfected cells in comparison to that of mock transfected cells. The fluorescence level was evaluated in triplicate and the assays were performed three times with similar results. ${ }^{*} \mathrm{P}<0.05,{ }^{* *} \mathrm{P}<0.01$. revealed a significant inhibition of cell migration/invasion through the matrix coated pore membrane dish in the cells transfected with siFAT1 (Fig. 4F).

\section{Discussion}

FAT1 is considered to be a tumor suppressor gene based on the evidence in Drosophila and the results of $\mathrm{CGH}$ studies (1,7-9). In addition, FAT1 indirectly regulates cell proliferation; Hou et al demonstrated that the inhibition of FAT1 expression promotes cell proliferation whereas the overexpression of FAT1 suppresses proliferation in vascular muscle cells (10). Therefore, the inhibition of FAT1 expression was expected to increase the cell proliferation in the OSCCs as well. In this study, the silencing of FAT1 mRNA had limited effect on the proliferation of OSCC cell lines. Thus, the role of FAT cadherin in cell proliferation of OSCCs might be limited in comparison to that of other oncogenes and/or tumor suppressor genes.

FAT1 was previously suggested to be a tumor suppressor gene in human (8). Nevertheless, the current study revealed the expression of FAT1 in most of the OSCC cases and cell lines. Interestingly, the expression and localization patterns of FAT1 in OSCCs were different from those of normal cells. The results from the previous and this study suggest that FAT1 does not act as the 'pure' tumor suppressor gene while the difference of the localization of FAT1 might be associated with the cancerous phenotypes.

The deletion of this gene product may play a pivotal role in the metamorphosis into characteristic carcinoma cells which includes the loss of cell to cell adhesion, cell polarity and morphology. One of the typical roles of FAT1 gene products is maintaining polarity and morphology in cooperation with stress fibers, Ena/Vasp and $\beta$-catenin that bind to the cytoplasmic domain of FAT1 (2,3). Moreover, FAT1 itself has a nuclear localization signal sequence and the translocation of its cytoplasmic domain to the nucleus might result in binding to Atrophin, while also inducing cell migration and polarity (18-20). This study demonstrated that the silencing of FAT1 resulted in a revere reduction in cell to cell adhesion, and change in cell morphology associated with the disorganized localization of $\beta$-catenin. This result suggested that the deletion of FAT1 contributes to the 'cancerous' disorganized morphology of OSCCs. Therefore, limited cell to cell adhesion could result in tumors that can be easily disseminated and metastasize, as observed with the deletion of E-cadherin (21-24).

Nevertheless, the silencing of FAT1 suppressed the mobility of the OSCCs in the wound healing assay and cell migration/ invasion assay. An inhibited expression of FAT1 in those experiments was also associated with the drastically disorganized localization of $\beta$-catenin. This phenomenon could indicate that FAT1 binds to the cytoplasmic domain of $\beta$-catenin as a member of cell membrane-cadherin complexes; while the deletion of FAT1 resulted in the diffuse localization of $\beta$-catenin in the cytoplasm and nucleus. Several studies have described the nuclear translocation of $\beta$-catenin, which is associated with LEF-1/TCF, thus resulting in an up-regulation the transcription of oncogenes including MYCBP $(25,26)$. On the other hand, the absence of the organized complex of FAT1 
and $\beta$-catenin might result in the loss of both cell polarity as well as migration ability.

The tumor from two cases in the current series lacked FAT1 expression. Both demonstrated less invasiveness and a good prognosis, although there were not enough FAT1negative tumor cases for perform statistical evaluations. Further study should therefore be carried out in order to reveal the clinical characteristics of FAT1-negative OSCCs.

The findings of this study suggest that the inhibition of FAT1 in OSCCs might result in the suppression of tumor migration/invasion and it could also be linked to a better prognosis. FAT1 is thus considered to be a potential target for the development of new molecular therapeutic strategies to improve the prognosis of OSCCs.

\section{Acknowledgement}

We thank to Dr Yuki Tanaka (Integrated Center for Scientific research, Ehime University) for her kind help in cell-free protein synthesis, Mr. Masachika Shudo (Integrated Center for Scientific Research, Ehime University) for his technical assistance in LSM. Also we thank Ms. Yumiko Fukuda and Mr. Takeshi Kiyoi for their technical assistance. This study was supported by Grant-in-Aid for Scientific Research Program for Graduate Student by Ehime University, Grantin-Aid for Scientific Research (C) (no. 21592557) by the Japan Society for the Promotion of Science, and in part by a Grant-in-Aid for Scientific Research from the Ministry of Education, Culture, Sports, Science and Technology of Japan (Program for Enhancing Systematic Education in Graduate School).

\section{References}

1. Dunne J, Hanby AM, Poulsom R, Jones TA, Sheer D, Chin WG, Da SM, Zhao Q, Beverley PC and Owen MJ: Molecular cloning and tissue expression of FAT, the human homologue of the Drosophila fat gene that is located on chromosome 4q34-q35 and encodes a putative adhesion molecule. Genomics 30: 207-223, 1995.

2. Tanoue T and Takeichi M: Mammalian Fatl cadherin regulates actin dynamics and cell-cell contact. J Cell Biol 165: 517-528, 2004.

3. Moeller MJ, Soofi A, Braun GS, Li X, Watzl C, Kriz W and Holzman LB: Protocadherin FAT1 binds Ena/VASP proteins and is necessary for actin dynamics and cell polarization. EMBO J 23: 3769-3779, 2004.

4. Huber TB and Benzing T: The slit diaphragm: a signaling platform to regulate podocyte function. Curr Opin Nephrol Hypertens 14: 211-216, 2005.

5. Blair IP, Chetcuti AF, Badenhop RF, Scimone A, Moses MJ, Adams LJ, Craddock N, Green E, Kirov G, Owen MJ, Kwok JB, Donald JA, Mitchell PB and Schofield PR: Positional cloning, association analysis and expression studies provide convergent evidence that the cadherin gene FAT contains a bipolar disorder susceptibility allele. Mol Psychiatry 11: 372-383, 2006.

6. Light KJ, Miller AL, Doughty CJ, Joyce PR, Olds RJ and Kennedy MA: FAT and bipolar affective disorder. Mol Psychiatry 12: 899-900, 2007.

7. Watson KL, Justice RW and Bryant PJ: Drosophila in cancer research: the first fifty tumor suppressor genes. J Cell Sci (Suppl) 18: 19-33, 1994.
8. Nakaya K, Yamagata HD, Arita N, Nakashiro KI, Nose M, Miki T and Hamakawa H: Identification of homozygous deletions of tumor suppressor gene FAT in oral cancer using CGH-array. Oncogene 26: 5300-5308, 2007.

9. Chosdol K, Misra A, Puri S, Srivastava T, Chattopadhyay P, Sarkar C, Mahapatra AK and Sinha S: Frequent loss of heterozygosity and altered expression of the candidate tumor suppressor gene 'FAT' in human astrocytic tumors. BMC Cancer 9: 5, 2009.

10. Hou R, Liu L, Anees S, Hiroyasu S and Sibinga NE: The Fat1 cadherin integrates vascular smooth muscle cell growth and migration signals. J Cell Biol 173: 417-429, 2006.

11. Tanoue $\mathrm{T}$ and Takeichi M: New insights into Fat cadherins. J Cell Sci 118: 2347-2353, 2005.

12. Schreiner D, Muller K and Hofer HW: The intracellular domain of the human protocadherin hFat1 interacts with Homer signalling scaffolding proteins. FEBS Lett 580: 5295-5300, 2006.

13. Castillejo-Lopez C, Arias WM and Baumgartner S: The fat-like gene of Drosophila is the true orthologue of vertebrate fat cadherins and is involved in the formation of tubular organs. J Biol Chem 279: 24034-24043, 2004.

14. Ma D, Yang CH, McNeill H, Simon MA and Axelrod JD: Fidelity in planar cell polarity signalling. Nature 421: 543-547, 2003.

15. Hou R and Sibinga NE: Atrophin proteins interact with the Fat1 cadherin and regulate migration and orientation in vascular smooth muscle cells. J Biol Chem 284: 6955-6965, 2009.

16. Nagae S, Tanoue $T$ and Takeichi M: Temporal and spatial expression profiles of the Fat 3 protein, a giant cadherin molecule, during mouse development. Dev Dyn 236: 534-543, 2007.

17. Klosek SK, Nakashiro K, Hara S, Goda H and Hamakawa H: Stat 3 as a molecular target in RNA interference-based treatment of oral squamous cell carcinoma. Oncol Rep 20: 873-878, 2008.

18. Fanto M, Clayton L, Meredith J, Hardiman K, Charroux B, Kerridge $\mathrm{S}$ and McNeill $\mathrm{H}$ : The tumor-suppressor and cell adhesion molecule Fat controls planar polarity via physical interactions with Atrophin, a transcriptional co-repressor. Development 130: 763-774, 2003.

19. Magg T, Schreiner D, Solis GP, Bade EG and Hofer HW: Processing of the human protocadherin Fat1 and translocation of its cytoplasmic domain to the nucleus. Exp Cell Res: 307 100-108, 2005.

20. Matakatsu H and Blair SS: Separating the adhesive and signaling functions of the Fat and Dachsous protocadherins. Development 133: 2315-2324, 2006.

21. Schipper JH, Frixen UH, Behrens J, Unger A, Jahnke K and Birchmeier W: E-cadherin expression in squamous cell carcinomas of head and neck: inverse correlation with tumor dedifferentiation and lymph node metastasis. Cancer Res 51: 6328-6337, 1991.

22. Nakayama S, Sasaki A, Mese H, Alcalde RE, Tsuji T and Matsumura T: The E-cadherin gene is silenced by CpG methylation in human oral squamous cell carcinomas. Int J Cancer 93: 667-673, 2001.

23. Campos RS, Lopes A, Guimaraes GC, Carvalho AL and Soares FA: E-cadherin, MMP-2, and MMP-9 as prognostic markers in penile cancer: analysis of 125 patients. Urology 67: 797-802, 2006

24. Lopes FF, da Costa Miguel MC, Pereira AL, da Cruz MC, de Almeida Freitas R, Pinto LP and de Souza LB: Changes in immunoexpression of E-cadherin and beta-catenin in oral squamous cell carcinoma with and without nodal metastasis. Ann Diagn Pathol 13: 22-29, 2009.

25. Behrens J, von Kries JP, Kuhl M, Bruhn L, Wedlich D, Grosschedl R and Birchmeier W: Functional interaction of beta-catenin with the transcription factor LEF-1. Nature 382: 638-642, 1996.

26. Jung HC and Kim K: Identification of MYCBP as a beta-catenin/ LEF-1 target using DNA microarray analysis. Life Sci 77: 12491262,2005 . 